\title{
Evidence-based guideline recommendations on multiparametric magnetic resonance imaging in the diagnosis of clinically significant prostate cancer: A Cancer Care Ontario updated clinical practice guideline
}

\author{
Masoom A. Haider, MD'; Judy Brown, PhD², Jospeh L.K. Chin, MD'; Nathan Perlis, MD'; Nicola Schieda, MD'; \\ Andrew Loblaw, $M D^{6}$
}

'Sinai Health System and University of Toronto, Joint Department of Medical Imaging, Toronto, ON, Canada; 2Program in Evidence-based Care, Ontario Health (Cancer Care Ontario), McMaster University, Hamilton ON, Canada; ${ }^{3}$ London Health Sciences Centre, Victoria Hospital, London, ON, Canada; ${ }^{4}$ Cancer Clinical Research Unit, Princess Margaret Cancer Centre, Toronto, ON, Canada; ${ }^{5}$ Department of Radiology, University of Ottawa, Ottawa, ON, Canada; 'Sunnybrook Health Sciences Centre, Toronto, ON, Canada

Cite as: Haider MA, Brown J, Chin JLK, et al. Evidence-based guideline recommendations on multiparametric magnetic resonance imaging in the diagnosis of clinically significant prostate cancer: A Cancer Care Ontario updated clinical practice guideline. Can Urol Assoc J 2022;16(2):16-23. http://dx.doi.org/10.5489/cuaj.7425

See related commentary on page 15

Note, this guideline was developed by the CCO and is endorsed by the CUA; however, it did not undergo the standard CUA guideline development and review process.

\section{Abstract}

Introduction: This clinical practice guideline is based on a systematic review to assess the use of multiparametric magnetic resonance imaging ( $\mathrm{mpMRI})$ in the diagnosis of clinically significant prostate cancer (csPCa) for biopsy-naive men and men with a prior negative transrectal ultrasound-guided systematic biopsy (TRUS-SB) at elevated risk

Methods: The methods of the clinical practice guideline included searches to September of 2020 of MEDLINE, EMBASE, and the Cochrane Central Register of Controlled Trials. Internal and external reviews were conducted.

Results: The recommendations are:

Recommendation 1: For biopsy-naive patients at elevated risk of csPCa, mpMRI is recommended prior to biopsy in patients who are candidates for curative management with suspected clinically localized prostate cancer.

- If the mpMRI is positive, mpMRI-targeted biopsy (TB) and TRUS-SB should be performed together to maximize detection of $\mathrm{CsPCa}$.

- If the mpMRI is negative, consider forgoing any biopsy after discussion of the risks and benefits with the patient as part of shared decision-making and ongoing followup.
Recommendation 2: In patients who had a prior negative TRUSSB and demonstrate a high risk of having csPCa in whom curative management is being considered:

- mpMRI should be performed.

- If the mpMRI is positive, targeted biopsy should be performed. Concomitant TRUS-SB can be considered depending on the patient's risk profile and time since prior TRUS-SB biopsy.

- If the mPMRI is negative, consider forgoing a TRUS-SB only after discussion of the risks and benefits with the patient as part of shared decision-making and ongoing followup. Recommendation 3: mpMRI should be performed and interpreted in compliance with the current Prostate Imaging Reporting \& Data System (PI-RADS) guidelines.

\section{Introduction}

Prostate cancer is the most common cancer among Canadian men, excluding non-melanoma skin cancers, and is the third leading cause of death in Canadian male cancer patients. ${ }^{1}$ In most clinical practices, the current standard for diagnosing clinically significant prostate cancer (csPCa) in biopsy-naive men at risk is transrectal ultrasound (TRUS)-guided systematic biopsy (TRUS-SB) of 10-12 cores. ${ }^{2}$ Transperineal systematic biopsy may also performed but is less commonly applied in Canada. Because TRUS-SB systematically samples areas from the prostate and not a specific imaged target, this approach has been shown to lead to over-detection of clinically insignificant prostate cancer (cisPCa) ${ }^{3}$ and can miss csPCa. ${ }^{4}$

Over the past several years, there has been growing use of multiparametric magnetic resonance imaging (mpMRI) as a non-invasive tool to diagnose and localize csPCa. mpMRI followed by targeted biopsy (mpMRI-TB), particularly in men with prior negative biopsy, may be considered in the detection of csPCa, as per the Ontario provincial guidelines previ- 
ously published. ${ }^{5}$ However, at the time of the writing of that guideline, there was a paucity of high-quality data supporting the use of mpMRI-TB in biopsy-naive men. In addition, there have been no Canadian guidelines published that address the minimum acceptable standards in the acquisition, interpretation, and reporting of mpMRI or the minimal acceptable standards for performance of mpMRI-TB. The guidelines sought to address this latter issue primarily through expert opinion.

The Working Group (WG) guideline authors (with expertise in diagnostic imaging, radiation oncology, urology, and health research methodology), in association with the Program in Evidence-based Care (PEBC) of Ontario Health (Cancer Care Ontario) and the mpMRI in Prostate Cancer Guideline Development Group (GDG) conducted an update of a systematic review to develop a clinical practice guideline to assess the use of mpMRI in the diagnosis of csPCa for biopsy-naive men and men with a prior negative TRUS-SB at elevated risk (according to prostate-specific antigen [PSA] levels and/or nomograms).

\section{Methods}

The systematic review will be published separately. Briefly, MEDLINE (May 2013 through September 1, 2020), EMBASE (May 2013 through September 1, 2020), the Cochrane Central Register of Controlled Trials (OVID CCTR: September 2020), and the Database of Abstracts of Reviews of Effects (OVID DARE: third quarter 2020) were searched for systematic reviews, review-based guidelines, original studies, and conference abstracts.

The report was assessed and approved by the PEBC Report Approval Panel (RAP), which consisted of two oncologists with expertise in clinical and methodological issues. Nine members of the mpMRI in the Diagnosis of Clinically Significant Prostate Cancer Expert Panel (EP) (a larger group of radiologists, urologists, and surgical oncologists of which the WG were selected) also reviewed and approved this report.

Following approval by the RAP and EP, a targeted peer review was conducted to obtain direct feedback on the draft report from a small number of specified content experts, and a professional consultation took place, intended to facilitate dissemination of the final guidance report to Ontario practitioners.

\section{Results}

\section{Literature search results}

Of the 3754 studies identified in the literature search, 36 studies from 39 publications ${ }^{6-44}$ met the inclusion criteria. The overall risk of bias of the studies ranged from low to high. ${ }^{45-47}$

\section{Internal and external review}

The summary of main RAP and EP comments and the WG's responses/modifications are shown in Table 1.

Responses were received by two targeted reviewers. Results of the feedback survey are summarized in Table 2. The main comments from targeted peer-reviewers and responses form the WG are summarized in Table 3.

The response rate for professional consultation was $6 \%$ (12 respondents). The results of the survey from the 12 participants are summarized in Table 4. The main comments from the professional consultation and the WG's responses are summarized in Table 5.

\section{Practice guidelines}

The finalized version of the report reflects feedback from the internal and external review processes, with final approval granted by the mpMRI in Prostate Cancer GDG. These guidelines apply to patients without contraindications to mpMRI (i.e., patients with MRI-incompatible medical devices).

\section{Recommendation 1}

For biopsy-naive patients at elevated risk of csPCa, mpMRI is recommended prior to biopsy in patients who are candidates for curative management with suspected clinically localized prostate cancer.

- If the mpMRI is positive, mpMRI-TB and TRUS-SB should be performed together to maximize detection of csPCa.

- If the mpMRI is negative, consider forgoing any biopsy after discussion of the risks and benefits with the patient as part of shared decision-making and ongoing followup.

\section{Key evidence for recommendation 1}

Twenty-three trials (all full-text publications) compared mpMRI with a reference standard $(n=5$, all cohort studies) or with TRUS-SB ( $n=18: 2$ randomized controlled trials [RCTs] and 16 cohort studies) for biopsy-naive men. The certainty of the aggregate study evidence for each comparison showed 14 of the 21 cohort studies to be at either low $^{6,32,40}$ or moderate $7,9,11,2,14,16,18,22,25,33,44$ risk of bias based on a GRADE approach. ${ }^{48}$ One of the RCTs was assessed to be at low risks of bias $^{20}$ and the other was assessed at being at unclear risk. ${ }^{30}$

- In the five studies where template transperineal mapping biopsy (TTMB) was the reference standard, mpMRI ranges were sensitivity 87-96\%, specificity 29-45\%, positive predictive values (PPVs) 46-65\%, and negative predictive values (NPVs) 76-92\%. ${ }^{6,17,18,25,38}$ Of these five studies, PROMIS was a prospective, multicenter trial (MCT). ${ }^{6}$ In this study, 


Main comments \\ The PRECISE data likely would not influence the recommendations, but they should be incorporated into the evidence discussion. This was a major trans-Canadian initiative, co-funded by the Ontario Institute for Cancer Research, whose goal was to influence funding for prostate MRI in Canada. \\ I have some serious concerns about the wording of Recommendation 2. In particular, the statement, "In patients who had a prior negative TRUS-SB and demonstrate a high or an increasing risk of having csPCa in whom curative management is being considered: mpMRI should be performed." The problem with this strategy is the risk of overdiagnosis.}

Table 1. Responses regarding main comments from the Report Approval Panel and Expert Pane

Treatment alternatives in Recommendation 1 should be expanded beyond surgery and radiation, (i.e., to include partial gland ablation and energybased technologies). The statement implying that radiation and surgery are the only curative options is outdated. Suggest including partial gland ablation as a treatment option. (This is not to endorse partial gland ablation, but only to acknowledge they are approved options that are often offered to patients).

Obviously, the issue of the role of systematic biopsies in men having targeted biopsy is not black and white. If the objective is to maximize diagnosis, they are clearly required. But another objective is to minimize morbidity and reduce number of cores. In the lower-risk patient, the NPV in the regions of the gland where the MRI is negative is sufficiently high $(90 \%)$ that systematic biopsies may be omitted. Therefore, I believe the concept of risk stratification as the basis for decision-making should be addressed in the document more than it is.

\section{Responses}

PRECISE trial results have been added in the discussion of the systematic review.

The principal role for MRI in biopsy-naive patients is complete biopsy avoidance to reduce the risk of overdiagnosis. This is the primary advantage of the strategy and produces the largest reduction in overdiagnosis. Once a decision to perform a biopsy is made because of a positive MRI, it is assumed there is also an intent to pursue curative intent therapy. mpMRI-TB combined with TRUS-SB in MRI-positive patients still allows for overall reduction in TRUS-SB in those patients who are mpMRInegative, with only a slight increase in cisPCa detection (8\%) while increasing CSPCa detection by $6 \%$.

Removed specification of radiation therapy and surgery leaving the door open to focal therapy or other curative intent therapies in the future.

We have not further delved into risk stratification, as this is an extensive and complex topic and beyond the scope of this document. A change has been made to the target population definition as follows:

"Patients with an elevated risk of csPCa (defined as International Society of Urologic Pathology [ISUP] grade group [GG] $\geq 2$ ), as estimated by available clinical information and tools, such as risk calculators and nomograms...."

cis: clinically insignificant; csPCa: clinically significant prostate cancer; mpMRI: multiparametric magnetic resonance imaging; NPV: negate predictive value; TB: target biopsy; TRUS-SB: transrectal ultrasound-guided systematic biopsy.

it was estimated that unnecessary biopsies could be reduced by up to $27 \%$. mpMRI was more sensitive (88\% vs. $48 \%$, 95\% confidence interval $[\mathrm{Cl}] 43-54$, $\mathrm{p}<0.0001)$ but less specific ( $45 \%$ vs. $99 \%, 95 \% \mathrm{Cl}$ 97-100, $\mathrm{p}<0.0001)$ than TRUS-SB in this study. ${ }^{6}$
- Two RCTs compared CSPCa detection rates of mpMRI-TB vs. TRUS-SB. ${ }^{20,30}$ Estimates for csPCa when combining the two RCTs showed increased detection favoring mpMRI by $18 \%(95 \% \mathrm{Cl} 5-32$, $\mathrm{p}=0.009$ ). Estimates for the two RCTs combined for

Table 2. Results from the targeted peer-reviewed questionnaire

\begin{tabular}{|c|c|c|c|c|c|}
\hline \multirow[b]{2}{*}{ Questions } & \multicolumn{5}{|c|}{ Reviewer ratings $(n=2)$} \\
\hline & $\begin{array}{l}\text { Lowest quality } \\
\text { (1) }\end{array}$ & (2) & (3) & (4) & $\begin{array}{l}\text { Highest quality } \\
\text { (5) }\end{array}$ \\
\hline Rate the guideline development methods. & 0 & 0 & 0 & 0 & 2 \\
\hline Rate the guideline presentation. & 0 & 0 & 0 & 2 & 0 \\
\hline Rate the guideline recommendations. & 0 & 0 & 0 & 2 & 0 \\
\hline Rate the completeness of reporting. & 0 & 0 & 0 & 0 & 2 \\
\hline $\begin{array}{l}\text { Does this document provide sufficient information to } \\
\text { inform your decisions? If not, what areas are missing? }\end{array}$ & 0 & 0 & 0 & 0 & 2 \\
\hline \multirow[t]{2}{*}{ Rate the overall quality of the guideline report. } & 0 & 0 & 0 & 1 & 1 \\
\hline & $\begin{array}{c}\text { Strongly } \\
\text { disagree (1) }\end{array}$ & (2) & Neutral (3) & (4) & $\begin{array}{l}\text { Strongly agree } \\
\text { (5) }\end{array}$ \\
\hline $\begin{array}{l}\text { I would make use of this guideline in my professional } \\
\text { decisions. }\end{array}$ & 0 & 0 & 0 & 1 & 1 \\
\hline I would recommend this guideline for use in practice. & 0 & 0 & 0 & 2 & 0 \\
\hline
\end{tabular}




\begin{tabular}{|c|c|}
\hline Main comments & Responses \\
\hline \multirow{4}{*}{$\begin{array}{l}\text { One thing that I was uncertain } \\
\text { of is the nature of a "negative } \\
\text { biopsy" (i.e., no prostate } \\
\text { cancer seen or does a } \\
\text { negative biopsy include GG } \\
1 \text { prostate cancer). It might } \\
\text { be worthwhile to make a } \\
\text { disclaimer that this guideline } \\
\text { is not addressing the use of } \\
\text { mpMRI for men diagnosed } \\
\text { with cisPCa on previous } \\
\text { biopsies. I wonder if a quick } \\
\text { sentence to clarify that may } \\
\text { ensure clinicians are not } \\
\text { expecting recommendations } \\
\text { on the use of mpMRI } \\
\text { in patients on active } \\
\text { surveillance. }\end{array}$} & We added a phrase: \\
\hline & $\begin{array}{l}\text { "Patients with an elevated risk of } \\
\text { csPCa (defined as ISUP GG } \geq 2 \text { ), } \\
\text { as estimated by available clinical } \\
\text { information and tools, such as } \\
\text { risk calculators and nomograms, } \\
\text { of who are A) biopsy-naive or B) } \\
\text { have had a prior negative TRUS- } \\
\text { SB, defined as no prostate cance } \\
\text { on biopsy of any grade group." }\end{array}$ \\
\hline & $\begin{array}{l}\text { A definition has been added } \\
\text { under qualifying statements for } \\
\text { Recommendation } 2 \text { : }\end{array}$ \\
\hline & $\begin{array}{l}\text { "Prior negative TRUS-SB is } \\
\text { defined as no cancer of any GG } \\
\text { on prior biopsy." }\end{array}$ \\
\hline & $\begin{array}{l}\text { s: clinically significant; ISUP GG: } \\
\text { JP] grade group; mpMRI: multiparametric } \\
\text { ectal ultrasound-guided systematic biopsy. }\end{array}$ \\
\hline
\end{tabular}

cisPCa showed decreased detection favoring mpMRI by $9 \%(95 \% \mathrm{Cl}-17-1, \mathrm{p}=0.03)$.

- In total, 16 cohort studies and the two RCTs mentioned above presented detection rates comparing mpMRI-TB to TRUS-SB. ${ }^{7,9,11,12,14,16,22-24,31-33,40,42-44}$ Estimates for csPCa showed increased detection favoring mpMRI-TB by $3 \%(95 \% \mathrm{Cl} 0-7, \mathrm{p}=0.03)$. For cisPCa, the estimate showed decreased detection favoring mpMRI by $8 \%(95 \% \mathrm{Cl}-11-5 \%$, $\mathrm{p}<0.00001)$.

- Of the above cohort studies examining mpMRI-TB vs. TRUS-SB, two were prospective MCTs. ${ }^{32,40} \mathrm{~A}$ paired diagnostic study (MRI-FIRST) enrolled 251 patients. ${ }^{32}$ Patients received both TRUS-SB and mpMRI-TB. There were no significant differences in the detection of csPCa in mpMRI-TB vs. TRUS-SB ( $32 \%$ vs. $30 \%$, $\mathrm{p}=0.225)$. However, mpMRI-TB detected significantly less cisPCa than TRUS-SB (6\% vs. $20 \%$, p<0.0001). Five percent of CsPCa was detected by TRUS-SB that was missed by mpMRI-TB and $8 \%$ was detected by mpMRI-TB and missed by TRUS-SB. Thus, detection of csPCa was improved by combining TRUS-SB and
mpMRI-TB. ${ }^{32}$ Another prospective MCT enrolled 646 men to receive MPMRI followed by TRUS-SB and in-bore MPRI-TB. ${ }^{40}$ This study showed similar csPCa detection rates ( $25 \%$ vs. $23 \%, p=0.392)$; however, cisPCa was detected in significantly fewer patients by mpMRI-TB than in TRUS-SB (14\% vs. $25 \%, \mathrm{p}<0.0001)$. mpRI-TB enabled biopsy avoidance in $49 \%$ of patients while missing only 35 cases with csPCa. Meanwhile, TRUS-SB would have overdetected cisPCa in $20 \%$ of patients. ${ }^{40}$

Overall estimates for the studies comparing mpMRI-TB plus TRUS-SB to targeted biopsy alone showed $6 \%$ increased csPCa detection when combining the systematic and targeted biopsy $(95 \% \mathrm{Cl} 4-8, \mathrm{p}<0.00001)$ and $8 \%$ increased detection of cisPCa (95\% Cl 6-10, p<0.00001). ${ }^{9,11,12,14,16,31-33,39,40,42-44}$

\section{Justification for recommendation 1}

- The issue of how targeted biopsy alone should be interpreted in overall whole gland Gleason scoring has not been resolved in the care community. Targeted biopsy plus systematic biopsy is believed to be necessary if mpMRI is positive in biopsy-naive patients, as multifocality and positive biopsy in other regions not seen by mpMRI is important in clinical decision-making and treatment planning given the use of focal dose-escalation therapies. In addition, the risk of severe complications, such as hospital admission for urosepsis, does not increase when changing from targeted biopsy to targeted biopsy plus systematic biopsy, although the risk of less severe complications does increase.

- Multiple MCTs have shown a decrease in cisPCa detection rate without reduction in CsPCa detection rate when using mpMRI-TB compared with TRUS-SB.

- The principal value of mpMRI in biopsy-naive patients is biopsy avoidance, with up to a $49 \%$ reduction in biopsies if mpMRI-negative patients are not biopsied. ${ }^{40}$

- Although mpMRI may miss $8-24 \%$ of csPCa in individual patients, ${ }^{6,38}$ these mpMRI-negative patients can be surveilled clinically, while avoiding the disadvantages of TRUS-SB, such as over-diagnosis of cisPCa and

Table 4. Results from the profession consultation survey

\begin{tabular}{|c|c|c|c|c|c|}
\hline & \multicolumn{5}{|c|}{ Number 12 (5.9\%) } \\
\hline & $\begin{array}{l}\text { Lowest quality } \\
\text { (1) }\end{array}$ & (2) & (3) & (4) & $\begin{array}{l}\text { Highest quality } \\
\text { (5) }\end{array}$ \\
\hline \multirow[t]{2}{*}{ Rate the overall quality of the guideline report. } & 0 & 0 & 0 & 6 & 6 \\
\hline & $\begin{array}{c}\text { Strongly } \\
\text { disagree (1) }\end{array}$ & (2) & (3) & (4) & $\begin{array}{c}\text { Strongly agree } \\
\text { (5) }\end{array}$ \\
\hline $\begin{array}{l}\text { I would make use of this guideline in my } \\
\text { professional decisions. }\end{array}$ & 0 & 0 & 2 & 3 & 7 \\
\hline I would recommend this guideline for use in practice. & 0 & 1 & 0 & 5 & 6 \\
\hline
\end{tabular}




\begin{tabular}{|c|c|}
\hline Comments & Responses \\
\hline $\begin{array}{l}\text { I think some mention of PSA } \\
\text { is indicated (also maybe DRE } \\
\text { abnormalities) The report, at face } \\
\text { value, indicates that a normal } \\
\text { mpMRI should lead to a shared } \\
\text { decision but implies a biopsy is not } \\
\text { needed. I think this is very different } \\
\text { for a patient with a PSA of } 8 \text { vs. a } \\
\text { PSA of } 25 \text { (or a DRE abnormality } \\
\text { perhaps) or a very high PSA } \\
\text { density. I do not see these items } \\
\text { addressed. }\end{array}$ & $\begin{array}{l}\text { These points are well } \\
\text { taken; however, specific } \\
\text { recommendations on how } \\
\text { risk should be assessed are } \\
\text { difficult and beyond the } \\
\text { scope of this guideline. }\end{array}$ \\
\hline $\begin{array}{l}\text { Many studies are available to } \\
\text { estimate the number of biopsy } \\
\text { avoidance based on a negative } \\
\text { mpMRI result among the biopsy- } \\
\text { naive patients. }\end{array}$ & $\begin{array}{l}\text { There were reviews } \\
\text { identified that did not fully } \\
\text { meet our study criteria and, } \\
\text { thus, were not used (did not } \\
\text { separate biopsy-naive and } \\
\text { previously negative men } \\
\text { according to our inclusion } \\
\text { criteria). }\end{array}$ \\
\hline $\begin{array}{l}\text { More specific guidance on who } \\
\text { can apply bpMRI would also } \\
\text { be helpful; we have considered } \\
\text { switching to bpMRI to expedite } \\
\text { MRI exams, given our long wait- } \\
\text { times; however, we decided not } \\
\text { to, given our uncertainty about the } \\
\text { tradeoffs and the experience level } \\
\text { of our radiologists }\end{array}$ & $\begin{array}{l}\text { This is out of scope and will } \\
\text { have to come from further } \\
\text { discussions with Ministry/ } \\
\text { CCO. }\end{array}$ \\
\hline onance imaging: mp: multiparametric:PSA pros & 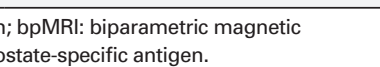 \\
\hline
\end{tabular}

complications, including urosepsis, urinary retention, hematuria, and rectal bleeding. The patients that gain the most in the biopsy-naive group are the mpMRInegative patients. The primary goal is safe avoidance of cisPCa detection (over-detection) in this cohort. If no biopsy is performed, it is essential that the patient and urologist commit to ongoing followup, given the risk of under-detection of csPCa by mpPMRI.

- mpMRI-TB combined with TRUS-SB in MRI-positive patients still allows for overall reduction in TRUSSB in those patients who are mpMRI-negative, with only a slight increase in cisPCa detection ( $8 \%$ ) while increasing csPCa detection by $5 \%$.

\section{Recommendation 2}

In patients who had a prior negative TRUS-SB and demonstrate a high risk of having CsPCa in whom curative management is being considered:

- mpMRI should be performed,

- If the mpMRI is positive, targeted biopsy should be performed. Concomitant TRUS-SB can be considered depending on the patients risk profile and time since prior TRUS-SB biopsy.

- If the mpMRI is negative, consider forgoing a TRUSSB only after discussion of the risks and benefits with the patient as part of shared decision-making and ongoing followup.

\section{Key evidence for recommendation 2}

Twenty-two trials (all full-text publications) compared mpMRI with a reference standard $(n=7)$ or with TRUS-SB $(n=15)$ for previously negative men. The certainty of the aggregate study evidence for each comparison showed 15 of the 22 studies to be at either low, $8,10,15,37$, moderate, ${ }^{7,12,16,21,22,25}$ or unclear ${ }^{17,19,27-29}$ risk of bias based on a GRADE approach. ${ }^{48}$

- Seven studies reported on the diagnostic accuracy of mpMRI for previously negative patients with sensitivities of $78-100 \%$, specificities of $30-100 \%$, PPVs of $36-100 \%$, and NPVs of $69-100 \% .^{18,19,25,27-29,37}$

- The overall improvement in csPCa detection rate for the 15 studies comparing mpMRI-TB alone to TRUS$\mathrm{SB}$ was $5 \%(95 \% \mathrm{Cl} 3-7, \mathrm{p}<0.0001)$, with a reduction of cisPCa detection of $7 \%(95 \% \mathrm{Cl} 4-9, \mathrm{p}<0.00001)$.

- The overall improvement in csPCa detection for the five cohort studies comparing mpMRI-TB plus TRUS-SB to mpMRI-TB alone was 5\% $(95 \% \mathrm{Cl} 2-8$, $\mathrm{p}=0.0005)$.

- The overall improvement across studies in csPCa detection for mpMRI-TB plus TRUS-SB compared with TRUS-SB alone was 11\% (95\% Cl 8-14, p<0.00001).

\section{Justification for recommendation 2}

- All the eligible studies show mpMRI-TB detected a higher number of csPCa when compared with TRUS-SB.

\section{Recommendation 3}

- $\quad$ mpMRI should be performed and interpreted in compliance with the current Prostate Imaging Reporting \& Data System (PI-RADS) guidelines (v2.1 as of summer 2020; see https://www.acr.org/Clinical-Resources/ Reporting-and-Data-Systems/PI-RADS).

- mpMRI-TB is recommended for MRI lesions with a PI-RADS score of 4 or 5 .

- mpMRI-TB or followup is recommended for MRI lesions with a PI-RADS score of 3 depending on the patient's risk profile.

- Biopsy avoidance should be considered when maximum PI-RADS score is 1 or 2 (see Recommendations 1 and 2).

- A structured mpMRI reporting template as recommended by the PI-RADS committee should be 
used (see https://www.acr.org/Clinical-Resources/ Reporting-and-Data-Systems/PI-RADS).

- When a targeted biopsy is being performed, a minimum of two cores should be taken per target, with recommendation of four cores for the index lesion. If multiple lesions are described on mpMRI, the biopsy operator may distribute the number of biopsies to keep a reasonable overall core count during the biopsy session.

- mpMRI interpretation and mpMRI-TB should be performed by experienced operators.

- A provincial quality assurance program should be developed. Until this is in place, practitioners should have some form of local quality assurance in place.

\section{Key evidence for recommendation 3}

- This recommendation is based on expert opinion and review of the PI-RADS committee guidelines, as well as the Standard Operating Procedure of the American Urological Association (AUA) (https://www.auanet. org/guidelines/mri-of-the-prostate-sop).

- Four cores per lesion have been performed in recent MCTs evaluating mpMRI but if one combines systematic biopsy and four cores/lesion in a patient with multiple mpMRI lesions, the core count will be unreasonable. Prior single-center studies have shown small incremental and diminishing increases in target biopsy yield as core count increases. ${ }^{49-51}$ For this reason, the operator is given discretion in the choice of number of cores per target for non-index lesions or when multiple lesions are present. mpMRI diagnostic performance varies by reader experience, as does mpMRI-TB performance. ${ }^{52}$

\section{Justification for recommendation 3}

- All the published studies demonstrating the performance of mpMRI involved diagnostic radiologists and biopsy operators with training and experience in performing mpMRI and mpMRI-TB. They all used defined five-point scoring schemes and, more recently, have used the PI-RADS v2 scoring scheme. To ensure similar performance in clinical practice, radiologists interpreting $\mathrm{mpMRI}$ and practitioners performing mpMRI-TB should have experience and demonstrate consistent performance levels.

\section{Implementation considerations}

Before mpMRI is used in clinical practice, radiologists who perform and interpret mpMRI and physicians treating PCa should be familiar with current PI-RADS prostate MRI minimum technical specifications and reporting standards. ${ }^{39}$ The patient care pathway in Ontario and the incorporation of mpMRI will need ongoing evaluation for its impact on patient care and outcomes.

The value of mpMRI cannot be realized without attention to quality assurance. Studies have demonstrated only moderate agreement in PI-RADS scoring among readers ${ }^{40,41}$ and a wide $\mathrm{Cl}$ for the PPVs of PI-RADS score $\geq 3$ (35\%, 95\% $\mathrm{Cl} 27-43 \%){ }^{42}$ There is currently no quality assurance program in place for mpMRI in Ontario or nationally. Quality standards or development of a quality assurance program is advisable before wide scale adoption of these recommendations occurs outside of centers with established expertise. Since prostate $\mathrm{mpMRI}$ and $\mathrm{mpMRI-TB}$ involve new technologies, skills, and education, knowledge transfer to practitioners should also be considered as part of implementation. In developing a local or provincial quality assurance program, metrics to consider collecting include: target yield (defined as the number of csPCa detected per lesion biopsied), stratified by PI-RADS score, and the number of false-negative $m p M R I$ (i.e., instances where mpMRI is reported as negative and a csPCa is diagnosed at TRUS-SB or prostatectomy). Changes may be required in biopsy collection and reporting at institutions that begin performing mpMRI-TB, where all targeted biopsy specimens are labeled and placed in a separate vial labelled with target number and location.

Although cost-effectiveness and resource allocation issues are beyond the scope of this PEBC guideline, the WG was sensitive to the fact that there are limited MRI resources in Canada. Further study into the resource implications of the implementation of these guidelines is required, especially in the biopsy-naive population addressed in Recommendation 1 . The lack of ready access to computer/software-aided fusion biopsy systems may require the use of cognitive fusion biopsy in many centers, which will require additional operator training, though the former are becoming more widely available. Cost savings from biopsy deferral in selected men choosing to forego TRUS-SB with negative mpMRI through shared decision-making could be considerable. Further cost savings may be realized through judicious use of biparametric MRI (bpMRI). The use of bpMRI in Canada is an attractive option to improve access and lower cost; however, this requires rigorous quality assurance, expertise, radiology/pathology feedback, and informed use from all stakeholders, including patients and physicians treating PCa.

The use of bpMRI, meaning omitting the dynamic contrast-enhanced MRI (DCEMRI), from mpMRI remains a controversial subject. This is being considered as an alternative to mpMRI, principally due to resource issues. By omitting DCEMRI, considerable savings in contrast agent cost and MRI time can be achieved. This is highly relevant in the context of the expected increase in volume of prostate MRI, with major implications on Canadian MRI capacity, once mpMRI becomes the anticipated standard of care in biopsy-naive patients. There are both single-center studies 
and meta-analyses data showing non-inferiority of bpMRI to mpMRI; ${ }^{46-49}$ however, concern remains regarding the retrospective nature of these studies and the potential increase in indeterminate (PI-RADS 3) interpretations using only bpMRI. Prospective MCT or trials comparing impact on decisionmaking and outcomes between bpMRI and mpMRI are lacking. For this reason, mpMRI is still recommended as the standard of care; however, given anticipated resource pressures, bpMRI can be performed at the discretion of the radiologist in centers that have demonstrated local bpMRI performance similar to mpMRI

It is expected that additional compelling evidence on the tradeoffs in diagnostic performance between mpMRI and bpMRI - its relationship to cost, safety, decision-making, and outcomes - will alter practice in the future. As the cost implications of implementing mpMRI in Ontario for biopsynaive patients may be prohibitive, the WG recognized that bpMRI may ease the financial burdens of performing MRI in this population and is a viable alternative to $\mathrm{mpMRI}$ if carefully monitored.

\section{Updating}

All PEBC documents are maintained and updated through an annual assessment review process (https://www.cancercareontario.ca/sites/ccocancercare/files/assets/CCOPEBCDARP.pdf).

Competing interests: The authors do not report any competing personal or financial interests related to this work.

\section{References}

1. Canadian cancer society's steering committee on cancer statistics. Canadian cancer statistics 2020. Toronto, on: Canadian cancer society; 2020. Available at: Http://www.Cancer.Ca/en/cancer-information/cancertype/prostate/statistics/?Region=on\#ixzz33sajdne4. Accessed March 12, 2021.

2. Mozer $P$, Rouprêt $M$, Le Cossec $C$, et al. First round of targeted biopsies using magnetic resonance imaging/ ultrasonography fusion compared with conventional transrectal ultrasonography-guided biopsies for the diagnosis of localised prostate cancer. BJU Int 2015;115:50-7. https://doi.org/10.1111/bju.12690

3. Schröder FH, Hugosson J, Roobol MJ, et al. Screening and prostate-cancer mortality in a randomized European study. N Engl J Med 2009;360:1320-8. https://doi.org/10.1056/nejmoa0810084

4. Sonn $G A$, Chang E, Natarajan $S$, et al. Value of targeted prostate biopsy using magnetic resonanceultrasound fusion in men with prior negative biopsy and elevated prostate-specific antigen. Research Support, N.I.H., Extramural Research Support, Non-U.S. Gov't. Eur Urol Apr 2014;65:809-15. https:// doi.org/10.1016/i.eururo.2013.03.025

5. Haider $M A$, Yao X, Loblaw A, et al. Evidence-based guideline recommendations on multiparametric magnetic resonance imaging in the diagnosis of prostate cancer: A Cancer Care Ontario clinical practice guideline. Can Urol Assoc J 2017;11:E1-7. htrps://doi.org/10.5489/cuaj.3968

6. Ahmed HU, El-Shater Bosaily A, Brown LC, et al. Diagnostic accuracy of multi-parametric MRI and TRUS biopsy in prostate cancer (PROMIS): A paired validating confirmatory study. Lancet 2017;389:815-22. https://doi.org/10.1016/S0140-6736(16)32401-1

7. Alberts AR, Schoots IG, Bokhorst $L P$, et al. Characteristics of prostate cancer found at fifth screening in the European randomized study of screening for prostate cancer Rotterdam: Can we selectively detect high-grade prostate cancer with upfront multivariable risk stratification and magnetic resonance imaging? Eur Urol 2017;73:353-60. https://doi.org/10.1016/i.eururo.2017.06.019
8. Arsov C, Rabenalt R, Blondin D, et al. Prospective randomized trial comparing magnetic resonance imaging (MRI)-guided in-bore biopsy to MRl-ultrasound fusion and transrectal ultrasound-guided prostate biopsy in patients with prior negative biopsies. Eur Urol 2015;68:713-20. https://doi.org/10.1016/i. eururo.2015.06.008

9. Baco E, Rud E, Eri LM, et al. A randomized, controlled trial to assess and compare the outcomes of two-core prostate biopsy guided by fused magnetic resonance and transrectal ultrasound images and traditional 12-core systematic biopsy. Eur Urol 2016;69:149-56. https://doi.org/10.1016/j.eururo.2015.03.041

10. Boesen L, Norgaard N, Logager V, et al. Multiparametric MRI in men with clinical suspicion of prostate cancer undergoing repeat biopsy: A prospective comparison with clinical findings and histopathology. Acta Radiol 2018;59:371-80. https://doi.org/10.1177/0284185117718400

11. Borkowetz A, Hadaschik B, Platzek I, et al. Prospective comparison of transperineal magnetic resonance imaging/Ultrasonography fusion biopsy and transrectal systematic biopsy in biopsy-naive patients. BJU Int 2018;121:53-60. https://doi.org/10.1111/bju.14017

12. Borkowetz A, Platzek I, Toma M, et al. Evaluation of prostate imaging reporting and data system classification in the prediction of tumor aggressiveness in targeted magnetic resonance imaging/ultrasound-fusion biopsy. Urol Int 2017;99:177-85. https://doi.org/10.1159/000477263

13. Brown LC, Ahmed HU, Faria R, et al. Multiparametric MRI to improve detection of prostate cancer compared with transrectal ultrasound-guided prostate biopsy alone: The PROMIS study. Health Tech Assess 2018;22:1-176. htrps://doi.org/10.3310/hta22390

14. Castellucci R, Linares Quevedo Al, Sanchez Gomez FJ, et al. Prospective, non-randomized study of diagnostic accuracy comparing prostate cancer detection by transrectal ultrasound-guided biopsy to magnetic resonance imaging with subsequent MRl-guided biopsy in biopsy-naive patients. Review. Minerva Urol Nefrol 2017;69:589-95. hittps://doi.org/10.23736/S0393-2249.17.02845-4

15. Exterkate L, Wegelin 0 , Barentsz J0, et al. Is there still a need for repeated systematic biopsies in patients with previous negative biopsies in the era of magnetic resonance imaging-targeted biopsies of the prostate? Eur Urol Oncol 2020;3:216-23. https://doi.org/10.1016/i.euo.2019.06.005

16. Filson CP, Natarajan S, Margolis DJ, et al. Prostate cancer detection with magnetic resonance-ultrasound fusion biopsy: The role of systematic and targeted biopsies. Cancer 2016;122:884-92. https://doi. org/10.1002/cncr.29874

17. Hansen N, Patruno G, Wadhwa K, et al. Magnetic resonance and ultrasound image fusion supported transperineal prostate biopsy using the Ginsburg protocol: Technique, learning points, and biopsy results. Eur Urol 2016;70:332-40. https://doi.org/10.1016/i.eururo.2016.02.064

18. Hansen NL, Barrett T, Kesch C, et al. Multicenter evaluation of magnetic resonance imaging supported transperineal prostate biopsy in biopsy-naive men with suspicion of prostate cancer. BJU Int 2018;122:409. https://doi.org/10.1111/bju.14049

19. Hansen NL, Kesch C, Barrett T, et al. Multicenter evaluation of targeted and systematic biopsies using magnetic resonance and ultrasound image-fusion guided transperineal prostate biopsy in patients with a previous negative biopsy. BJU Int 2017;120:631-8. https://doi.org/10.1111/bju.13711

20. Kasivisvanathan V, Rannikko AS, Borghi M, et al. MRl-targeted or standard biopsy for prostate-cancer diagnosis. N Engl J Med 2018;378:1767-77. https://doi.org/10.1056/neimoa1801993

21. Lian H, Zhuang J, Wang W, et al. Assessment of free-hand transperineal targeted prostate biopsy using multiparametric magnetic resonance imaging-transrectal ultrasound fusion in Chinese men with prior negative biopsy and elevated prostate-specific antigen. BMC Urol 2017;17:52. https://doi.org/10.1186/ s12894-017-0241-3

22. Mannaerts CK, Kaitazovic A, Lodeizen OAP, et al. The added value of systematic biopsy in men with suspicion of prostate cancer undergoing multiparametric MRl-targeted biopsy. Urol Oncol 2019;37:298e]9. https://doi.org/10.1016/j.urolonc.2019.01.005

23. Mariotti GC, Costa DN, Pedrosa I, et al. Magnetic resonance/transrectal ultrasound fusion biopsy of the prostate compared to systematic 12-core biopsy for the diagnosis and characterization of prostate cancer: Multi-institutional retrospective analysis of 389 patients. Urol Oncol 2016;34:416e9-14. https://doi. org/10.1016/i.urolonc.2016.04.008

24. Meng X, Rosenkrantz AB, Mendhiratta N, et al. Relationship between prebiopsy multiparametric magnetic resonance imaging (MRI), biopsy indication, and MRl-ultrasound fusion-targeted prostate biopsy outcomes. Eur Urol 2016;69:512-7. hittps://doi.org/10.1016/i.eururo.2015.06.005

25. Mortezavi A, Marzendorfer 0 , Donati $0 F$, et al. Diagnostic accuracy of multiparametric magnetic resonance imaging and fusion guided targeted biopsy evaluated by transperineal template saturation prostate biopsy for the detection and characterization of prostate cancer. J Urol 2018;200:309-18. hitps://doi. org/10.1016/i.juro.2018.02.067

26. Peltier $A$, Aoun $F$, Lemort $M$, et al. MRl-targeted biopsies versus systematic transrectal ultrasound guided biopsies for the diagnosis of localized prostate cancer in biopsy-naive men. BioMed Res Int 2015;2015:571708. https://doi.org/10.1155/2015/571708

27. Pepe P, Garufi A, Priolo G, et al. Can 3-tesla pelvic phased-array multiparametric MRI avoid unnecessary repeat prostate biopsy in patients with PSA<10 ng/ml? Clin Genitourin Cancer 2015;13:e27-30. https:// doi.org/10.1016/i.clgc.2014.06.013 
28. Pepe P, Garufi A, Priolo GD, et al. Is it time to perform only magnetic resonance imaging targeted cores? Our experience with 1032 men who underwent prostate biopsy. J Urol 2018;200:774-8. https://doi. org/10.1016/i.juro.2018.04.061

29. Pepe P, Garufi A, Priolo GD, et al. Multiparametric MRI/TRUS fusion prostate biopsy: Advantages of a transperineal approach. Anticancer Res 2017;37:3291-4. https://doi.org/10.21873/anticanres.11695

30. Porpiglia F, Manfredi M, Mele F, et al. Diagnostic pathway with multiparametric magnetic resonance imaging versus standard pathway: Results from a randomized, prospective study in biopsy-naive patients with suspected prostate cancer. Eur Urol 2017;72:282-8. https://doi.org/10.1016/i.eururo.2016.08.041

31. Preisser $F P$, Theissen L, Wenzel $M$, et al. Performance of combined magnetic resonance imaging/ultrasound fusion-guided and systematic biopsy of the prostate in biopsy-naive patients and patients with prior biopsies. Eur Urol Focus 2021;7:39-46. https://doi.org/10.1016/j.euf.2019.06.015

32. Rouviere 0 , Puech P, Renard-Penna R, et al. Use of prostate systematic and targeted biopsy on the basis of multiparametric MRI in biopsy-naive patients (MRI-first): A prospective, multicenter, paired diagnostic study. Lancet Oncol 2019;20:100-9. https://doi.org/10.1016/s1470-2045(18)30569-2

33. Sarkar D, Nandi D, Gangoli S, et al. The decision of targeted, systematic, or combined biopsy in a biopsy naive patient for the diagnosis of prostate cancer, can be made on the basis of multiparametric magnetic resonance imaging. J Clin Urol 2020;13:198-204. https://doi.org/10.1177/2051415819889552

34. Say R. MRl-ultrasound fusion targeted biopsy in men with prior negative prostate biopsy for prostate cancer. 2016. Thesis. Available at: https://elischolar.library.yale.edu/cgi/viewcontent. cgi?article=2078\&context=ymtdl. Accessed Dec, 3, 2021.

35. Sidana A, Watson MJ, George AK, et al. Fusion prostate biopsy outperforms 12-core systematic prostate biopsy in patients with prior negative systematic biopsy: A multi-institutional analysis. Urol Oncol 2018;36:341el-7. https://doi.org/10.1016/j.urolonc.2018.04.002

36. Simmons LAM, Kanthabalan A, Arya M, et al. The picture study: Diagnostic accuracy of multiparametric MRI in men requiring a repeat prostate biopsy. Br J Cancer 2017;116:1159-65. https://doi.org/10.1038/ bic. 2017.57

37. Simmons LAM, Kanthabalan A, Arya M, et al. Accuracy of transperineal targeted prostate biopsies, visual estimation, and image fusion in men needing repeat biopsy in the picture trial. J Urol 2018;200:1227-34 https://doi.org/10.1016/i.juro.2018.07.001

38. Thompson JE, van Leeuwen PJ, Moses D, et al. The diagnostic performance of multiparametric magnetic resonance imaging to detect significant prostate cancer. J Urol 2016;195:1428-35. https://doi. org/10.1016/i.juro.2015.10.140

39. Tontilia PP, Lantto J, Paakko E, et al. Prebiopsy multiparametric magnetic resonance imaging for prostate cancer diagnosis in biopsy-naive men with suspected prostate cancer based on elevated prostate-specific antigen values: Results from a randomized, prospective, blinded, controlled trial. Eur Urol 2016;69:41925. https://doi.org/10.1016/i.eururo.2015.05.024

40. van der Leest $M$, Cornel $E$, Israel $B$, et al. Head-to-head comparison of transrectal ultrasound-guided prostate biopsy vs. multiparametric prostate resonance imaging with subsequent magnetic resonance-guided biopsy in biopsy-naive men with elevated prostate-specific antigen: A large, prospective, multicenter clinical study. Eur Urol 2019;75:570-8. https://doi.org/10.1016/i.eururo.2018.11.023
41. Wegelin 0 , Exterkate $L$, van der Leest $M$, et al. The future trial: A multicenter, randomized, controlled trial on target biopsy techniques based on magnetic resonance imaging in the diagnosis of prostate cancer in patients with prior negative biopsies. Eur Urol 2019;75:582-90. https://doi.org/10.1016/i. eururo.2018.11.040

42. Westhoff N, Baessler B, von Hardenberg J, et al. Systematic prostate biopsy still matters: A comprehensive analysis of MRI/TRUS-fusion targeted prostate biopsies across different indications. Urol Oncol 2019;37:678-87. hitps://doi.org/10.1016/i.urolonc.2019.07.004

43. Zalesky M, Stejskal J, Minarik I, et al. Cancer detection rates and inter-examiner variability of MRI/TRUS fusion targeted biopsy and systematic transrectal biopsy. Biomed Pap Med Fac Univ Palacky Olomouc Czech Repub 2020;164:314-9. htrps://doi.org/10.5507/bp.2019.050

44. Zhang $Q$, Wang $W$, Zhang $B$, et al. Comparison of free-hand transperineal mpMRI/TRUS fusion-guided biopsy with transperineal 12-core systematic biopsy for the diagnosis of prostate cancer: A single-center, prospective study in China. Int Urol Nephrol 2017;49:439-48. https://doi.org/10.1007/s11255016-1484-8

45. Sterne JA, Hernán $M A$, Reeves $B C$, et al. Robins-l: A tool for assessing risk of bias in non-randomized studies of interventions. BMJ 2016;355:14919. https://doi.org/10.1136/bmi.i4919

46. Whiting PF, Rutjes AWS, Westwood ME, et al. Quadas-2: A revised tool for the quality assessment of diagnostic accuracy studies. Ann Int Med 2011;155:529-36. https://doi.org/10.7326/0003-4819155-8-201110180-00009

47. Higgins JP, Alman DG, Gotzsche PC, et al. The Cochrane collaboration's tool for assessing risk of bias in randomized trials. BMJ 2011;343:d5928. https://doi.org/10.1136/bmi.d5928

48. Schünemann HJ, Mustafa R, Brozek J, et al. GRADE guidelines: 16. GRADE evidence to decision frameworks for tests in clinical practice and public health. J Clin Epidemiol 2016;76:89-98. https://doi. org/10.1016/i.jlinepi.2016.01.032

49. Zhang M, Milot L, Khalvati F, et al. Value of increasing biopsy cores per target with cognitive MRItargeted transrectal us prostate biopsy. Radiology 2019;291:83-9. https://doi.org/10.1148/ radiol.2019180712

50. Dimitroulis $P$, Rabenalt $R$, Nini $A$, et al. Multiparametric magnetic resonance imaging/ultrasound fusion prostate biopsy — are 2 biopsy cores per magnetic resonance imaging lesion required? J Urol 2018;200:1030-4. https://doi.org/10.1016/i.juro.2018.05.002

51. Kenigsberg AP, Renson A, Rosenkrantz AB, et al. Optimizing the number of cores targeted during prostate magnetic resonance imaging fusion target biopsy. Eur Urol Oncol 2018;1:418-25. https://doi. org/10.1016/i.euo.2018.09.006

52. Stabile A, Giganti F, Kasivisvanathan V, et al. Factors influencing variability in the performance of multiparametric magnetic resonance imaging in detecting clinically significant prostate cancer: A systematic literature review. Eur Urol Oncol 2020;3:145-67. https://doi.org/10.1016/i.euo.2020.02.005

Correspondence: Dr. Judy Brown, Program in Evidence-based care, Ontario Health (Cancer Care Ontario), Hamilton, 0N, Canada; browni@mcmaster.ca 